\title{
Characterization of Temperatures Associated to Schrödinger Operators with Initial Data in Morrey Spaces
}

\author{
Qiang Huang and Chao Zhang*
}

\begin{abstract}
Let $\mathcal{L}$ be a Schrödinger operator of the form $\mathcal{L}=-\Delta+V$ acting on $L^{2}\left(\mathbb{R}^{n}\right)$ where the nonnegative potential $V$ belongs to the reverse Hölder class $B_{q}$ for some $q \geq n$. Let $L^{p, \lambda}\left(\mathbb{R}^{n}\right), 0 \leq \lambda<n$ denote the Morrey space on $\mathbb{R}^{n}$. In this paper, we will show that a function $f \in L^{2, \lambda}\left(\mathbb{R}^{n}\right)$ is the trace of the solution of $\mathbb{L} u:=u_{t}+\mathcal{L} u=0$, $u(x, 0)=f(x)$, where $u$ satisfies a Carleson-type condition

$$
\sup _{x_{B}, r_{B}} r_{B}^{-\lambda} \int_{0}^{r_{B}^{2}} \int_{B\left(x_{B}, r_{B}\right)}|\nabla u(x, t)|^{2} d x d t \leq C<\infty .
$$

Conversely, this Carleson-type condition characterizes all the $\mathbb{L}$-carolic functions whose traces belong to the Morrey space $L^{2, \lambda}\left(\mathbb{R}^{n}\right)$ for all $0 \leq \lambda<n$. This result extends the analogous characterization found by Fabes and Neri in 8 for the classical BMO space of John and Nirenberg.
\end{abstract}

\section{Introduction and statement of the main result}

In Harmonic Analysis, to study a (suitable) function $f(x)$ on $\mathbb{R}^{n}$ is to consider a harmonic function on $\mathbb{R}_{+}^{n+1}$ which has the boundary value as $f(x)$. A standard choice for such a harmonic function is the Poisson integral $e^{-t \sqrt{-\Delta}} f(x)$ and one recovers $f(x)$ when letting $t \rightarrow 0^{+}$, where $\Delta=\sum_{i=1}^{n} \partial_{x_{i}}^{2}$ is the Laplace operator. In other words, one obtains $u(x, t)=e^{-t \sqrt{-\Delta}} f(x)$ as the solution of the equation

$$
\begin{cases}\partial_{t t} u+\Delta u=0 & \text { if } x \in \mathbb{R}^{n}, t>0 \\ u(x, 0)=f(x) & \text { if } x \in \mathbb{R}^{n}\end{cases}
$$

This approach is intimately related to the study of singular integrals. In [22], the authors studied the classical case $f \in L^{p}\left(\mathbb{R}^{n}\right), 1 \leq p \leq \infty$.

It is well known that the BMO space, i.e., the space of functions of bounded mean oscillation, is natural substitution to study singular integral at the end-point space $L^{\infty}\left(\mathbb{R}^{n}\right)$.

Received June 21, 2018; Accepted November 12, 2018.

Communicated by Duy-Minh Nhieu.

2010 Mathematics Subject Classification. 42B35, 42B37, 35J10, 47F05.

Key words and phrases. Dirichlet problem, heat equation, Schrödinger operators, Morrey space, Carleson measure, reverse Hölder inequality.

*Corresponding author. 
A celebrated theorem of Fefferman and Stein [10] states that a BMO function is the trace of the solution of $\partial_{t t} u+\Delta u=0, u(x, 0)=f(x)$, whenever $u$ satisfies

$$
\sup _{x_{B}, r_{B}} r_{B}^{-n} \int_{0}^{r_{B}} \int_{B\left(x_{B}, r_{B}\right)}|t \nabla u(x, t)|^{2} \frac{d x d t}{t} \leq C<\infty,
$$

where $\nabla=\left(\nabla_{x}, \partial_{t}\right)=\left(\partial_{1}, \ldots, \partial_{n}, \partial_{t}\right)$. Conversely, Fabes, Johnson and Neri [7] showed that condition above characterizes all the harmonic functions whose traces are in $\mathrm{BMO}\left(\mathbb{R}^{n}\right)$ in 1976. The study of this topic has been widely extended to more general operators such as elliptic operators and Schrödinger operators (instead of the Laplacian), for more general initial data spaces and for domains other than $\mathbb{R}^{n}$ such as Lipschitz domains. For these generalizations, see $[1,5,8,9,14,17,20$.

In 8, Fabes and Neri further generalized the above characterization to caloric functions (temperature), that is the authors proved that a BMO function $f$ is the trace of the solution of

$$
\begin{cases}\partial_{t} u-\Delta u=0 & \text { if } x \in \mathbb{R}^{n}, t>0 \\ u(x, 0)=f(x) & \text { if } x \in \mathbb{R}^{n}\end{cases}
$$

whenever $u$ satisfies

$$
\sup _{x_{B}, r_{B}} r_{B}^{-n} \int_{0}^{r_{B}^{2}} \int_{B\left(x_{B}, r_{B}\right)}\left|\nabla_{x} u(x, t)\right|^{2} d x d t \leq C<\infty,
$$

and, conversely, the condition (1.2) characterizes all the carolic functions whose traces are in $\operatorname{BMO}\left(\mathbb{R}^{n}\right)$. The authors in [15] explored more information, related to harmonic functions and carolic functions, about this subject.

In this paper, we consider a similar characterization in Moerry space $L^{p, \lambda}\left(\mathbb{R}^{n}\right)$. It is known that $L^{p, 0}\left(\mathbb{R}^{n}\right)=L^{p}\left(\mathbb{R}^{n}\right)$ and $L^{p, \lambda}\left(\mathbb{R}^{n}\right)=\mathcal{C}^{p, \lambda}\left(\mathbb{R}^{n}\right) / \mathbb{C}$ for $0 \leq \lambda<n$, where $\mathcal{C}^{p, \lambda}\left(\mathbb{R}^{n}\right)$ denote the Campanato space. When $\lambda=n, \mathcal{C}^{p, n}\left(\mathbb{R}^{n}\right)=\operatorname{BMO}\left(\mathbb{R}^{n}\right)$. So, Morrey spaces were proposed to be intermediate function spaces between $L^{p}$ space and BMO space. For more information about Morrey spaces, see [26]. The main aim of this article is to study a similar characterization to (1.1) for the Schrödinger operator with some conditions on its potentials and boundary values in Morrey spaces. To be precise, let us consider the Schrödinger operator

$$
\mathcal{L}=-\Delta+V(x) \quad \text { on } L^{2}\left(\mathbb{R}^{n}\right), n \geq 3
$$

We assume that $V$ is a nonnegative potential, not identically zero and that $V \in B_{q}$ for some $q \geq n / 2$, which by definition means that $V \in L_{\text {loc }}^{q}\left(\mathbb{R}^{n}\right), V \geq 0$, and there exists a constant $C>0$ such that the reverse Hölder inequality

$$
\left(\frac{1}{|B|} \int_{B} V(y)^{q} d y\right)^{1 / q} \leq \frac{C}{|B|} \int_{B} V(y) d y
$$


holds for all balls $B$ in $\mathbb{R}^{n}$.

The operator $\mathcal{L}$ is a self-adjoint operator on $L^{2}\left(\mathbb{R}^{n}\right)$. Hence $\mathcal{L}$ generates the $\mathcal{L}$-heat semigroup

$$
\mathcal{T}_{t} f(x)=e^{-t \mathcal{L}} f(x)=\int_{\mathbb{R}^{n}} \mathcal{H}_{t}(x, y) f(y) d y, \quad f \in L^{2}\left(\mathbb{R}^{n}\right), t>0 .
$$

From the Feynman-Kac formula, it is well-known that the semigroup kernels $\mathcal{H}_{t}(x, y)$ of the operators $e^{-t \mathcal{L}}$ satisfies

$$
0 \leq \mathcal{H}_{t}(x, y) \leq h_{t}(x-y)
$$

for all $x, y \in \mathbb{R}^{n}$ and $t>0$, where

$$
h_{t}(x)=(4 \pi t)^{-n / 2} e^{-|x|^{2} /(4 t)}
$$

is the kernel of the classical heat semigroup $\left\{T_{t}\right\}_{t>0}=\left\{e^{t \Delta}\right\}_{t>0}$ on $\mathbb{R}^{n}$. For the classical heat semigroup associated with Laplacian, see [21]. In this article, we consider the parabolic Schrödinger differential operators

$$
\mathbb{L}=\partial_{t}+\mathcal{L}, \quad t>0, x \in \mathbb{R}^{n},
$$

see, for instance, $11,23,25$ and references therein. For $f \in L^{p}\left(\mathbb{R}^{n}\right), 1 \leq p<\infty$, it is well known that $u(x, t)=e^{-t \mathcal{L}} f(x), t>0, x \in \mathbb{R}^{n}$, is a solution to the heat equation

$$
\mathbb{L} u=\partial_{t} u+\mathcal{L} u=0 \quad \text { in } \mathbb{R}_{+}^{n+1}
$$

with the boundary data $f \in L^{p}\left(\mathbb{R}^{n}\right), 1 \leq p<\infty$. The equation $\mathbb{L} u=0$ is interpreted in the weak sense via a sesquilinear form, that is, $u \in W_{\text {loc }}^{1,2}\left(\mathbb{R}_{+}^{n+1}\right)$ is a weak solution of $\mathbb{L} u=0$ if it satisfies

$$
\begin{aligned}
& \int_{\mathbb{R}_{+}^{n+1}} \nabla_{x} u(x, t) \cdot \nabla_{x} \psi(x, t) d x d t-\int_{\mathbb{R}_{+}^{n+1}} u(x, t) \partial_{t} \psi(x, t) d x d t \\
+ & \int_{\mathbb{R}_{+}^{n+1}} V u \psi d x d t=0, \quad \forall \psi \in C_{0}^{1}\left(\mathbb{R}_{+}^{n+1}\right) .
\end{aligned}
$$

In the sequel, we call such a function $u$ an $\mathbb{L}$-carolic function associated to the operator $\mathbb{L}$.

In [25], the authors proved that the conclusion gotten by E. Fabes and U. Neri in 8 can be proved in the Schrödinger case. In [20], the authors considered the results in [7] in the case of Poisson integrals of Schrödinger operators with Morrey traces. As mentioned above, we are interested in deriving the characterization of the solution to the heat equation $\mathbb{L} u=0$ in $\mathbb{R}_{+}^{n+1}$ with boundary values in Morrey spaces. Recall that Morrey spaces were 
introduced in 1938 by C. Morrey [18] to consider the regularity problems of solutions to PDEs. For every $1 \leq p<\infty$ and $\lambda \in[0, n)$, the Morrey spaces $L^{p, \lambda}\left(\mathbb{R}^{n}\right)$ are defined as

$$
L^{p, \lambda}\left(\mathbb{R}^{n}\right)=\left\{f \in L_{\mathrm{loc}}^{p}\left(\mathbb{R}^{n}\right): \sup _{x \in \mathbb{R}^{n}, r>0} r^{-\lambda} \int_{B(x, r)}|f(y)|^{p} d y<\infty\right\} .
$$

This is a Banach space with respect to the norm

$$
\|f\|_{L^{p, \lambda}\left(\mathbb{R}^{n}\right)}=\left(\sup _{x \in \mathbb{R}^{n}, r>0} r^{-\lambda} \int_{B(x, r)}|f(y)|^{p} d y\right)^{1 / p}<\infty .
$$

Moreover, for every $1 \leq p<\infty$ and $\lambda>0$, the Campanato spaces $C^{P, \lambda}\left(\mathbb{R}^{n}\right)$ are defined as

$$
C^{p, \lambda}\left(\mathbb{R}^{n}\right)=\left\{f \in L_{\mathrm{loc}}^{p}\left(\mathbb{R}^{n}\right):\|f\|_{C^{p, \lambda}\left(\mathbb{R}^{n}\right)}<\infty\right\}
$$

with the Campanato seminorm being given by

$$
\|f\|_{C^{p, \lambda}\left(\mathbb{R}^{n}\right)}=\left(\sup _{x \in \mathbb{R}^{n}, r>0} r^{-\lambda} \int_{B(x, t)}\left|f(y)-f_{B(x, r)}\right|^{p} d y\right)^{1 / p}<\infty
$$

where $f_{B(x, r)}$ denotes the average value of $f$ on the ball $B(x, r)$. And when $\lambda \in[0, n)$, $\mathcal{C}^{p, \lambda}\left(\mathbb{R}^{n}\right) / \mathbb{C}=L^{p, \lambda}\left(\mathbb{R}^{n}\right)$. Specially, when $\lambda=0, \mathcal{C}^{p, 0}\left(\mathbb{R}^{n}\right) / \mathbb{C}=L^{p, 0}\left(\mathbb{R}^{n}\right)=L^{p}\left(\mathbb{R}^{n}\right)$.

Next, we introduce a new function class on the upper half plane $\mathbb{R}_{+}^{n+1}$.

Definition 1.1. Suppose $V \in B_{q}$ for some $q \geq n$ and $0 \leq \lambda<n$. We say that, a $C^{1_{-}}$ functions $u(x, t)$ defined on $\mathbb{R}_{+}^{n+1}$ belongs to the class $\operatorname{TL}_{\mathcal{L}}^{\lambda}\left(\mathbb{R}_{+}^{n+1}\right)$, if $u(x, t)$ is the solution of $\mathbb{L} u=0$ in $\mathbb{R}_{+}^{n+1}$ such that

$$
\|u\|_{\mathrm{TL}_{\mathcal{L}}^{\lambda}\left(\mathbb{R}_{+}^{n+1}\right)}^{2}=\sup _{x_{B}, r_{B}} r_{B}^{-\lambda} \int_{0}^{r_{B}^{2}} \int_{B\left(x_{B}, r_{B}\right)}|\nabla u(x, t)|^{2} d x d t<\infty,
$$

where $\nabla=\left(\nabla_{x}, \partial_{t}\right)$.

The following theorem is the main result of this article.

Theorem 1.2. Suppose $V \in B_{q}$ for some $q \geq n$ and $0 \leq \lambda<n$, then we have

(1) if $f \in L^{2, \lambda}\left(\mathbb{R}^{n}\right)$, then the function $u=e^{-t \mathcal{L}} f \in \mathrm{TL}_{\mathcal{L}}^{\lambda}\left(\mathbb{R}_{+}^{n+1}\right)$ and

$$
\|u\|_{\mathrm{TL}_{\mathcal{L}}^{\lambda}\left(\mathbb{R}_{+}^{n+1}\right)} \leq C\|f\|_{L^{2, \lambda}\left(\mathbb{R}^{n}\right)}
$$

(2) if $u \in \mathrm{TL}_{\mathcal{L}}^{\lambda}\left(\mathbb{R}_{+}^{n+1}\right)$, then there exists some $f \in L^{2, \lambda}\left(\mathbb{R}^{n}\right)$ such that $u=e^{-t \mathcal{L}} f$, and

$$
\|f\|_{L^{2, \lambda}\left(\mathbb{R}^{n}\right)} \leq C\|u\|_{\mathrm{TL}_{\mathcal{L}}^{\lambda}\left(\mathbb{R}_{+}^{n+1}\right)}
$$

with some constant $C>0$ independent of $u$ and $f$. 
We should mention that for the Schrödinger operator $\mathcal{L}$ in $\sqrt{1.3}$, an important property of the $B_{q}$ class, proved in [12, Lemma 3], assures that the condition $V \in B_{q}$ also implies $V \in B_{q+\epsilon}$ for some $\epsilon>0$ and that the $B_{q+\epsilon}$ constant of $V$ is controlled in terms of the one of $B_{q}$ membership. This in particular implies $V \in L_{\text {loc }}^{q}\left(\mathbb{R}^{n}\right)$ for some $q$ strictly greater than $n / 2$. However, in general the potential $V$ can be unbounded and does not belong to $L^{p}\left(\mathbb{R}^{n}\right)$ for any $1 \leq p \leq \infty$. As a model example, we could take $V(x)=|x|^{2}$. Moreover, as noted in [19], if $V$ is any nonnegative polynomial, then $V$ satisfies the stronger condition

$$
\max _{x \in B} V(x) \leq \frac{C}{|B|} \int_{B} V(y) d y
$$

which implies $V \in B_{q}$ for every $q \in(1, \infty)$ with a uniform constant.

This article is organized as follows. In Section 2, we recall some preliminary results including the kernel estimates of the heat semigroup related with $\mathcal{L}$, and prove some lemmas and certain properties of $\mathbb{L}$-carolic functions. In Section 3 , we will prove our main result, Theorem 1.2 ,

Throughout the article, the letters " $c$ " and " $C$ " will denote (possibly different) constants which are independent of the essential variables.

\section{Basic properties of the heat semigroups of Schrödinger operators}

In this section, we begin by recalling some basic properties of the nonnegative potential $V$ under the assumption (1.4) and the kernel estimates of the heat semigroup related with $\mathcal{L}$.

It follows from Lemmas 1.2 and 1.8 in [19] that there is a constant $C_{0}$ such that for a nonnegative Schwartz class function $\varphi$ there exists a constant $C$ such that

$$
\int_{\mathbb{R}^{n}} \varphi_{t}(x-y) V(y) d y \leq \begin{cases}C t^{-1}(\sqrt{t} / \rho(x))^{\delta} & \text { for } t \leq \rho(x)^{2}, \\ C(\sqrt{t} / \rho(x))^{C_{0}+2-n} & \text { for } t>\rho(x)^{2},\end{cases}
$$

where $\varphi_{t}(x)=t^{-n / 2} \varphi(x / \sqrt{t}), \delta=2-n / q>0$, and the critical radii function $\rho(x ; V)=\rho(x)$ above are determined by the function

$$
\rho(x)=\sup \left\{r>0: \frac{1}{r^{n-2}} \int_{B(x, r)} V(y) d y \leq 1\right\} .
$$

For the heat kernel $\mathcal{H}_{t}(x, y)$ of the semigroup $e^{-t \mathcal{L}}$, we have the following estimates.

Lemma 2.1. (see [6]) Suppose $V \in B_{q}$ for some $q>n / 2$. For every $N>0$, there exists a constant $C_{N}$ such that for every $x, y \in \mathbb{R}^{n}, t>0$,

(i) $0 \leq \mathcal{H}_{t}(x, y) \leq C_{N} t^{-n / 2} e^{-|x-y|^{2} /(c t)}\left(1+\frac{\sqrt{t}}{\rho(x)}+\frac{\sqrt{t}}{\rho(y)}\right)^{-N}$, and 
(ii) $\left|\partial_{t} \mathcal{H}_{t}(x, y)\right| \leq C_{N} t^{-(n+2) / 2} e^{-|x-y|^{2} /(c t)}\left(1+\frac{\sqrt{t}}{\rho(x)}+\frac{\sqrt{t}}{\rho(y)}\right)^{-N}$.

In fact, with the same computation as in the proof of [6. Proposition 4], we have

$$
\left|t^{m} \partial_{t}^{m} \mathcal{H}_{t}(x, y)\right| \leq C_{N} t^{-n / 2} e^{-|x-y|^{2} /(c t)}\left(1+\frac{\sqrt{t}}{\rho(x)}+\frac{\sqrt{t}}{\rho(y)}\right)^{-N} .
$$

Lemma 2.2. [5, Lemma 3.8] Suppose $V \in B_{q}$ for some $q>n$. Let $\beta=1-n / q$. For every $N>0$, there exist constants $C=C_{N}>0$ and $c>0$ such that for all $x, y \in \mathbb{R}^{n}$ and $t>0$, the $\mathcal{L}$-Heat semigroup kernels $\mathcal{H}_{t}(x, y)$, associated to $e^{-t \mathcal{L}}$, satisfy the following estimates:

(i) $\left|\nabla_{x} \mathcal{H}_{t}(x, y)\right|+\left|t \nabla_{x} \partial_{t} \mathcal{H}_{t}(x, y)\right| \leq C t^{-(n+1) / 2} e^{-|x-y|^{2} /(c t)}\left(1+\frac{\sqrt{t}}{\rho(x)}+\frac{\sqrt{t}}{\rho(y)}\right)^{-N}$;

(ii) for $|h|<|x-y| / 4$,

$$
\left|\nabla_{x} \mathcal{H}_{t}(x+h, y)-\nabla_{x} \mathcal{H}_{t}(x, y)\right| \leq C\left(\frac{|h|}{\sqrt{t}}\right)^{\beta} t^{-(n+1) / 2} e^{-|x-y|^{2} /(c t)}
$$

(iii) there is some $\delta>1$ such that

$$
\left|\sqrt{t} \nabla_{x} e^{-t \mathcal{L}}(1)(x)\right| \leq C \min \left\{\left(\frac{\sqrt{t}}{\rho(x)}\right)^{\delta},\left(\frac{\sqrt{t}}{\rho(x)}\right)^{-N}\right\} .
$$

We now recall a local behavior of solutions to $\partial_{t} u+\mathcal{L} u=0$, which was proved in 24 , Lemma 3.3], see it also in [11, Lemma 3.2]. We define parabolic cubes of center $(x, t)$ and radius $r$ by $B_{r}(x, t):=\left\{(y, s) \in \mathbb{R}^{n} \times \mathbb{R}_{+}:|y-x|<r, t-r^{2}<s \leq t\right\}=B(x, r) \times\left(t-r^{2}, t\right]$. And for every $(x, t),(y, s) \in \mathbb{R}^{n} \times(0, \infty)$, we define the parabolic metric: $|(x, t)-(y, s)|=$ $\max \left\{|x-y|,|s-t|^{1 / 2}\right\}$.

Lemma 2.3. 24, Lemma 3.3] Suppose $0 \leq V \in L_{\text {loc }}^{q}\left(\mathbb{R}^{n}\right)$ for some $q>n / 2$. Let $u$ be a weak solution of $\mathbb{L} u=0$ in the parabolic cube $B_{r_{0}}\left(x_{0}, t_{0}\right)$. Then there exists a constant $C=C_{n}>0$ such that

$$
\sup _{B_{r_{0} / 4}\left(x_{0}, t_{0}\right)}|u(x, t)| \leq C\left(\frac{1}{r_{0}^{n+2}} \int_{B_{r_{0} / 2}\left(x_{0}, t_{0}\right)}|u(x, t)|^{2} d x d t\right)^{1 / 2} .
$$

\section{Proof of the main theorem}

In this section, we will give the proof of Theorem 1.2 . First, we need make some preparations. 
Lemma 3.1. For every $u \in \mathrm{TL}_{\mathcal{L}}^{\lambda}\left(\mathbb{R}_{+}^{n+1}\right)$ and for every $k \in \mathbb{N}$, there exists a constant $C_{k, n}>0$ such that

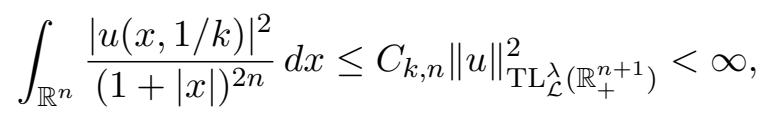

hence $u(x, 1 / k) \in L^{2}\left((1+|x|)^{-2 n} d x\right)$. Therefore for all $k \in \mathbb{N}, e^{-t \mathcal{L}}(u(\cdot, 1 / k))(x)$ exists everywhere in $\mathbb{R}_{+}^{n+1}$.

Proof. Since $u \in C^{1}\left(\mathbb{R}_{+}^{n+1}\right)$, it reduces to show that for every $k \in \mathbb{N}$,

$$
\int_{|x| \geq 1} \frac{|u(x, 1 / k)-u(x /|x|, 1 / k)|^{2}}{(1+|x|)^{2 n}} d x \leq C_{k, n}\|u\|_{\mathrm{TL}_{\mathcal{L}}^{\lambda}\left(\mathbb{R}_{+}^{n+1}\right)}^{2 n}<\infty .
$$

To do this, we write

$$
\begin{aligned}
u(x, 1 / k)-u(x /|x|, 1 / k)= & {[u(x, 1 / k)-u(x,|x|)]+[u(x,|x|)-u(x /|x|,|x|)] } \\
& +[u(x /|x|,|x|)-u(x /|x|, 1 / k)] .
\end{aligned}
$$

Let

$$
I=\int_{|x| \geq 1} \frac{|u(x, 1 / k)-u(x,|x|)|^{2}}{(1+|x|)^{2 n}} d x, \quad I I=\int_{|x| \geq 1} \frac{|u(x,|x|)-u(x /|x|,|x|)|^{2}}{(1+|x|)^{2 n}} d x
$$

and

$$
I I I=\int_{|x| \geq 1} \frac{|u(x /|x|,|x|)-u(x /|x|, 1 / k)|^{2}}{(1+|x|)^{2 n}} d x .
$$

For $|x| \geq 1$ and $t>0$, let $r^{2}=t / 4$. We use Lemma 2.3 for $\partial_{t} u$ and Schwarz's inequality to obtain

$$
\begin{aligned}
\left|\partial_{t} u(x, t)\right| & \leq C\left(\frac{1}{r^{n+2}} \int_{t-r^{2}}^{t} \int_{B(x, r)}\left|\partial_{s} u(y, s)\right|^{2} d y d s\right)^{1 / 2} \\
& \leq C\left(\frac{1}{t^{(n+2) / 2}} \int_{t-r^{2}}^{t} \int_{B(x, \sqrt{t} / 2)}\left|\partial_{s} u(y, s)\right|^{2} d y d s\right)^{1 / 2} \\
& \leq C t^{-\frac{1}{2}-\frac{n-\lambda}{4}}\left(\frac{1}{|B(x, \sqrt{t})|^{\lambda / n}} \int_{0}^{t} \int_{B(x, \sqrt{t})}\left|\partial_{s} u(y, s)\right|^{2} d y d s\right)^{1 / 2} \\
& \leq C t^{-\frac{1}{2}-\frac{n-\lambda}{4}}\|u\|_{\mathrm{TL}_{\mathcal{L}}^{\lambda}\left(\mathbb{R}_{+}^{n+1}\right)}
\end{aligned}
$$

which gives

$$
|u(x, 1 / k)-u(x,|x|)|=\left|\int_{1 / k}^{|x|} \partial_{t} u(x, t) d t\right| \leq C\left(|x|^{\frac{1}{2}-\frac{n-\lambda}{4}}-k^{-\frac{1}{2}+\frac{n-\lambda}{4}}\right)\|u\|_{\mathrm{TL}_{\mathcal{L}}^{\lambda}\left(\mathbb{R}_{+}^{n+1}\right)} .
$$


It follows that

$$
\begin{aligned}
I+I I I & \leq C\|u\|_{\mathrm{TL}_{\mathcal{L}}^{\lambda}\left(\mathbb{R}_{+}^{n+1}\right)}^{2} \int_{|x| \geq 1} \frac{1}{(1+|x|)^{2 n}}\left(|x|^{\frac{1}{2}-\frac{n-\lambda}{4}}-k^{\frac{n-\lambda}{4}-\frac{1}{2}}\right) d x \\
& \leq C(k, n)\|u\|_{\mathrm{TL}_{\mathcal{L}}^{\lambda}\left(\mathbb{R}_{+}^{n+1}\right)}^{2} .
\end{aligned}
$$

For the term $I I$, we have that for any $x \in \mathbb{R}^{n}$,

$$
u(x,|x|)-u(x /|x|,|x|)=\int_{1}^{|x|} D_{r} u(r \omega,|x|) d r, \quad x=|x| \omega .
$$

Let $B=B(0,1)$ and $2^{m} B=B\left(0,2^{m}\right)$. Note that for every $m \in \mathbb{N}$, we have

$$
\begin{aligned}
\int_{2^{m} B \backslash 2^{m-1} B}\left|\int_{1}^{|x|}\right| D_{r} u(r \omega,|x|)|d r|^{2} d x & =\int_{2^{m-1}}^{2^{m}} \int_{|\omega|=1}\left|\int_{1}^{\rho} D_{r} u(r \omega, \rho) d r\right|^{2} \rho^{n-1} d \omega d \rho \\
& \leq 2^{m n-m} \int_{2^{m-1}}^{2^{m}} \int_{|\omega|=1} \int_{1}^{2^{m}}\left|D_{r} u(r \omega, \rho)\right|^{2} d r d \omega d \rho \\
& \leq 2^{m n-m} \int_{2^{m-1}}^{2^{m}} \int_{2^{m} B \backslash B}\left|\nabla_{y} u(y, t)\right|^{2}|y|^{1-n} d y d t \\
& \leq 2^{m n-m} \int_{2^{m-1}}^{2^{m}} \int_{2^{m} B}\left|\nabla_{y} u(y, t)\right|^{2} d y d t,
\end{aligned}
$$

which gives

$$
\begin{aligned}
& \int_{2^{m} B \backslash 2^{m-1} B}|u(x,|x|)-u(x /|x|,|x|)|^{2} d x \\
\leq & C 2^{m n-m+m \lambda}\left(\frac{1}{\left|2^{m} B\right|^{\lambda / n}} \int_{0}^{2^{2 m}} \int_{2^{m} B}\left|\nabla_{y} u(y, t)\right|^{2} d y d t\right) \\
\leq & C 2^{(n+\lambda) m-m}\|u\|_{\mathrm{TL}_{\mathcal{L}}^{\lambda}\left(\mathbb{R}_{+}^{n+1}\right)}^{2} .
\end{aligned}
$$

Therefore,

$$
I I \leq C \sum_{m=1}^{\infty} \frac{1}{2^{2 n m}} \int_{2^{m} B \backslash 2^{m-1} B}|u(x,|x|)-u(x /|x|,|x|)|^{2} d x \leq C\|u\|_{\left.\mathrm{TL}_{\mathcal{L}^{\lambda}}^{\lambda} \mathbb{R}_{+}^{n+1}\right)}^{2} .
$$

Combining estimates of $I, I I$ and $I I I$, we have obtained (3.1).

Note that by Lemma 2.1. if $V \in B_{q}$ for some $q \geq n / 2$, then the semigroup kernels $\mathcal{H}_{t}(x, y)$, associated to $e^{-t \mathcal{L}}$, decay faster than any power of $1 /|x-y|$. Hence, for all $k \in \mathbb{N}$, $e^{-t \mathcal{L}}(u(\cdot, 1 / k))(x)$ exists everywhere in $\mathbb{R}_{+}^{n+1}$. This completes the proof.

Lemma 3.2. For every $u \in \mathrm{TL}_{\mathcal{L}}^{\lambda}\left(\mathbb{R}_{+}^{n+1}\right)$, we have that for every $k \in \mathbb{N}$,

$$
u(x, t+1 / k)=e^{-t \mathcal{L}}(u(\cdot, 1 / k))(x), \quad x \in \mathbb{R}^{n}, t>0 .
$$


Proof. The lemma can be proved by the same discussion as the proof of Lemma 3.2 in 5 and the proof of Lemma 3.3 in [25].

We recall that the classical Carleson measure is closely related to the space $\operatorname{BMO}\left(\mathbb{R}^{n}\right)$. In 25], the authors considered another similar Carleson measure which was called 2Carleson measure. Here, we need to consider a similar Carleson measure. We say that a measure $\mu$ defined on $\mathbb{R}_{+}^{n+1}$ is a $(2, \lambda)$-Carleson measure if there is a positive constant $c$ such that for each ball $B$, with radius $r_{B}$, in $\mathbb{R}^{n}$,

$$
\mu(\widehat{B}) \leq c|B|^{\lambda / n},
$$

where $\widehat{B}=\left\{(x, t): x \in B, 0 \leq t \leq r_{B}^{2}\right\}$ is the 2-tent over $B$. The smallest bound $c$ in (3.3) is defined to be the norm of $\mu$, and is denoted by \|\|$\mu\|\|_{(2, \lambda) \text { car }}$. When $\lambda=n$, it is coincided with the 2-Carleson measure in 25]. By using this measure, we will estimate the term $\partial_{t} e^{-t \mathcal{L}} f(x)$ in Morrey space. Precisely, for any $k \in \mathbb{N}$, we set

$$
u_{k}(x, t)=u(x, t+1 / k) .
$$

Following a similar argument as in [7, Lemma 1.4], we have the following lemma.

Lemma 3.3. For every $u \in \operatorname{TL}_{\mathcal{L}}^{\lambda}\left(\mathbb{R}_{+}^{n+1}\right)$, there exists a constant $C>0$ (depending only on $n$ ) such that for all $k \in \mathbb{N}$,

$$
\sup _{x_{B}, r_{B}} r_{B}^{-\lambda} \int_{0}^{r_{B}^{2}} \int_{B\left(x_{B}, r_{B}\right)}\left|\partial_{t} u_{k}(x, t)\right|^{2} d x d t \leq C\|u\|_{\mathrm{TL}_{\mathcal{L}}^{\lambda}\left(\mathbb{R}_{+}^{n+1}\right)}^{2}<\infty .
$$

Proof. Let $B=B\left(x_{B}, r_{B}\right)$. If $r_{B}^{2} \geq 1 / k$, then letting $s=t+1 / k$, it follows that

$$
\begin{aligned}
|B|^{-\lambda / n} \int_{0}^{r_{B}^{2}} \int_{B}\left|\partial_{t} u(x, t+1 / k)\right|^{2} d x d t & \leq C|B|^{-\lambda / n} \int_{0}^{\left(2 r_{B}\right)^{2}} \int_{2 B}\left|\partial_{s} u(x, s)\right|^{2} d x d s \\
& \leq C\|u\|_{\mathrm{TL}_{\mathcal{L}}^{\lambda}\left(\mathbb{R}_{+}^{n+1}\right)}^{2}<\infty .
\end{aligned}
$$

If $r_{B}^{2}<1 / k$, then it follows from Lemma 2.3 for $\partial_{t} u(x, t+1 / k)$ and a similar argument as in 3.2 that

$$
\left|\partial_{t} u(x, t+1 / k)\right| \leq C\left(t+k^{-1}\right)^{-\frac{1}{2}+\frac{n-\lambda}{4}}\|u\|_{\mathrm{TL}_{\mathcal{L}}^{\lambda}\left(\mathbb{R}_{+}^{n+1}\right)} .
$$

Therefore,

$$
\begin{aligned}
& |B|^{-\lambda / n} \int_{0}^{r_{B}^{2}} \int_{B}\left|\partial_{t} u(x, t+1 / k)\right|^{2} d x d t \\
\leq & C|B|^{-\lambda / n}\|u\|_{\mathrm{TL}_{\mathcal{L}}^{\lambda}\left(\mathbb{R}_{+}^{n+1}\right)}^{2} \int_{0}^{r_{B}^{2}} \int_{B}\left(t+k^{-1}\right)^{-(1+(n-\lambda) / 2)} d x d t \\
\leq & C\|u\|_{\mathrm{TL}_{\mathcal{L}}^{\lambda}\left(\mathbb{R}_{+}^{n+1}\right)}^{2}\left(k^{1+(n-\lambda) / 2} r_{B}^{n-\lambda} \int_{0}^{r_{B}^{2}} 1 d t\right) \\
\leq & C\|u\|_{\mathrm{TL}_{\mathcal{L}}^{\lambda}\left(\mathbb{R}_{+}^{n+1}\right)}^{2}<\infty
\end{aligned}
$$


since $r_{B}^{2}<1 / k$.

By taking the supremum over all balls $B \subset \mathbb{R}^{n}$, we complete the proof of 3.4 .

Letting $f_{k}(x)=u(x, 1 / k), k \in \mathbb{N}$, it follows from Lemma 3.2 that

$$
u_{k}(x, t)=e^{-t \mathcal{L}} f_{k}(x), \quad x \in \mathbb{R}^{n}, t>0 .
$$

And it follows from Lemma 3.3 that

$$
\sup _{x_{B}, r_{B}} r_{B}^{-\lambda} \int_{0}^{r_{B}^{2}} \int_{B\left(x_{B}, r_{B}\right)}\left|\partial_{t} e^{-t \mathcal{L}} f_{k}(x)\right|^{2} d x d t \leq C\|u\|_{\mathrm{TL}_{\mathcal{L}}^{\lambda}\left(\mathbb{R}_{+}^{n+1}\right)}^{2} .
$$

Lemma 3.4. For every $u \in \operatorname{TL}_{\mathcal{L}}^{\lambda}\left(\mathbb{R}_{+}^{n+1}\right)$, there exists a constant $C>0$ independent of $k$ such that

$$
\left\|f_{k}\right\|_{\mathrm{L}^{2, \lambda}\left(\mathbb{R}^{n}\right)} \leq C\|u\|_{\mathrm{TL}_{\mathcal{L}}^{\lambda}\left(\mathbb{R}_{+}^{n+1}\right)}<\infty \quad \text { for any } k \in \mathbb{N} .
$$

Hence for all $k \in \mathbb{N}, f_{k}$ is uniformly bounded in $\mathrm{L}^{2, \lambda}\left(\mathbb{R}^{n}\right)$.

To prove Lemma 3.4 we need to establish the following Lemmas 3.5 and 3.6 .

Given a function $f \in L^{2}\left((1+|x|)^{-2 n} d x\right)$ and an $L^{2} \subset L^{2 n /(n+2)}$ function $g$ supported on a ball $B=B\left(x_{B}, r_{B}\right)$, for any $(x, t) \in \mathbb{R}_{+}^{n+1}$, set

$$
F(x, t)=t \partial_{t} e^{-t \mathcal{L}} f(x) \text { and } \quad G(x, t)=t \partial_{t} e^{-t \mathcal{L}}\left(I-e^{-r_{B}^{2} \mathcal{L}}\right) g(x) .
$$

Lemma 3.5. Suppose $f, g, F, G$ are as in (3.5). If $f$ satisfies

$$
\| \mu_{\nabla_{t}, f}||_{(2, \lambda) \operatorname{car}}^{2}=\sup _{x_{B}, r_{B}} r_{B}^{-\lambda} \int_{0}^{r_{B}^{2}} \int_{B\left(x_{B}, r_{B}\right)}\left|\partial_{t} e^{-t \mathcal{L}} f(x)\right|^{2} d x d t<\infty,
$$

then there exists a constant $C>0$ such that

$$
\int_{\mathbb{R}_{+}^{n+1}}|F(x, t) G(x, t)| \frac{d x d t}{t} \leq C|B|^{\lambda /(2 n)} \mid\left\|\mu_{\nabla_{t}, f}\right\|\left\|_{(2, \lambda) \operatorname{car}}\right\| g \|_{L^{2 n /(n+2)}(B)} .
$$

Proof. To prove (3.6), let us consider the square functions $\mathcal{S}(f)$ and $\mathcal{G}(f)$ given by

$$
\mathcal{S}(f)(x)=\left(\int_{0}^{\infty}\left|t \partial_{t} e^{-t \mathcal{L}} f(x)\right|^{2} d t\right)^{1 / 2}, \quad \mathcal{G}(f)(x)=\left(\int_{0}^{\infty}\left|\partial_{t} e^{-t \mathcal{L}} f(x)\right|^{2} d t\right)^{1 / 2} .
$$

By the standard spectral theory as in [6], we have the following identities:

$$
\|\mathcal{S}(f)\|_{2}=\frac{1}{2}\left\|\mathcal{L}^{-1 / 2} f\right\|_{2}
$$

and

$$
\|\mathcal{G}(f)\|_{2}=\frac{\sqrt{2}}{2}\left\|\mathcal{L}^{1 / 2} f\right\|_{2}
$$


In fact, let us denote by $d E(\lambda)$ the spectral resolution of the operator $\mathcal{L}$. Since $e^{-t \mathcal{L}}=$ $\int_{0}^{\infty} e^{-t \lambda} d E(\lambda)$, we have

$$
t \partial_{t} e^{-t \mathcal{L}}=\int_{0}^{\infty} t \lambda e^{-t \lambda} d E(\lambda)
$$

Then, for all $f \in L^{2}\left(\mathbb{R}^{n}\right)$, we have

$$
\begin{aligned}
\left\|t \partial_{t} e^{-t \mathcal{L}} f(x)\right\|_{L^{2}\left(\mathbb{R}_{+}^{n+1}, d x d t\right)}^{2} & =\int_{0}^{\infty} \int_{\mathbb{R}^{n}}\left|t \partial_{t} e^{-t \mathcal{L}} f(x)\right|^{2} d x d t=\int_{0}^{\infty}\left\langle\left(t \partial_{t} e^{-t \mathcal{L}}\right)^{2} f, f\right\rangle_{L^{2}\left(\mathbb{R}^{n}\right)} d t \\
& =\int_{0}^{\infty} \int_{0}^{\infty} t^{2} \lambda^{2} e^{-2 t \lambda} d t d E_{f, f}(\lambda)=\frac{1}{4}\left\|\mathcal{L}^{-1 / 2} f\right\|_{L^{2}\left(\mathbb{R}^{n}\right)}^{2},
\end{aligned}
$$

which gives the proof of $(3.7)$. And the proof of $(3.8)$ is similar.

Given a ball $B=B\left(x_{B}, r_{B}\right) \subset \mathbb{R}^{n}$ with radius $r_{B}$, we put

$$
T(B)=\left\{(x, t) \in \mathbb{R}_{+}^{n+1}: x \in B, 0<t<r_{B}^{2}\right\} .
$$

We then write

$$
\begin{aligned}
& \int_{\mathbb{R}_{+}^{n+1}}|F(x, t) G(x, t)| \frac{d x d t}{t} \\
= & \int_{T(2 B)}|F(x, t) G(x, t)| \frac{d x d t}{t}+\sum_{k=2}^{\infty} \int_{T\left(2^{k} B\right) \backslash T\left(2^{k-1} B\right)}|F(x, t) G(x, t)| \frac{d x d t}{t} \\
= & \mathrm{A}_{1}+\sum_{k=2}^{\infty} \mathrm{A}_{k} .
\end{aligned}
$$

Using the Hölder inequality, (3.7) and the $L^{2}-L^{n /(n+2)}$ boundedness of fractional integral operator $\mathcal{L}^{-1 / 2}$, we obtain

$$
\begin{aligned}
\mathrm{A}_{1} & \leq\left\|\left\{\int_{0}^{\left(2 r_{B}\right)^{2}}\left|\partial_{t} e^{-t \mathcal{L}} f(x)\right|^{2} d t\right\}^{1 / 2}\right\|\left\|_{L^{2}(2 B)}\right\| \mathcal{S}\left(I-e^{-r_{B}^{2} \mathcal{L}}\right) g \|_{L^{2}\left(\mathbb{R}^{n}\right)} \\
& \leq C r_{B}^{\lambda / 2} \mid\left\|\mu_{\nabla_{t}, f}\right\|\left\|_{(2, \lambda) \operatorname{car}}\right\| \mathcal{L}^{-1 / 2}\left(I-e^{-r_{B}^{2} \mathcal{L}}\right) g \|_{L^{2}\left(\mathbb{R}^{n}\right)} \\
& \leq C r_{B}^{\lambda / 2}\|\| \mu_{\nabla_{t}, f}\|\|_{(2, \lambda) \operatorname{car}}\|g\|_{L^{2 n /(n+2)}(B)} .
\end{aligned}
$$

Let us estimate $\mathrm{A}_{k}$ for $k=2,3, \ldots$ Observe that

$$
\begin{aligned}
\mathrm{A}_{k} \leq & \left\|\left\{\int_{0}^{\left(2^{k} r_{B}\right)^{2}}\left|\partial_{t} e^{-t \mathcal{L}} f(x)\right|^{2} d t\right\}^{1 / 2}\right\|_{L^{2}\left(2^{k} B\right)} \\
& \times\left\|\left\{\int_{0}^{\left(2^{k} r_{B}\right)^{2}}\left|t \partial_{t} e^{-t \mathcal{L}}\left(I-e^{-r_{B}^{2} \mathcal{L}}\right) g(x) \chi_{T\left(2^{k} B\right) \backslash T\left(2^{k-1} B\right)}(x, t)\right|^{2} d t\right\}^{1 / 2}\right\| \|_{L^{2}\left(2^{k} B\right)} \\
\leq & C\left(2^{k} r_{B}\right)^{\lambda / 2} \mid\left\|\mu_{\nabla_{t}, f}\right\|_{(2, \lambda) \operatorname{car}} \times \mathrm{B}_{k},
\end{aligned}
$$


where

$$
\mathrm{B}_{k}=\left\|\left\{\int_{0}^{\left(2^{k} r_{B}\right)^{2}}\left|t \partial_{t} e^{-t \mathcal{L}}\left(I-e^{-r_{B}^{2} \mathcal{L}}\right) g(x) \chi_{T\left(2^{k} B\right) \backslash T\left(2^{k-1} B\right)}(x, t)\right|^{2} d t\right\}^{1 / 2}\right\|_{L^{2}\left(2^{k} B\right)} .
$$

To estimate $\mathrm{B}_{k}$, we set

$$
\Psi_{t, s}(\mathcal{L}) h(y)=(t+s)^{2}\left(\left.\frac{d^{2} e^{-r \mathcal{L}}}{d r^{2}}\right|_{r=t+s} h\right)(y)
$$

Note that

$$
\left(I-e^{-r_{B}^{2} \mathcal{L}}\right) g=\int_{0}^{r_{B}^{2}} \mathcal{L} e^{-s \mathcal{L}} g d s
$$

By (2.1), we have

$$
\begin{gathered}
\mathrm{B}_{k} \leq C\left\|\left\{\int_{0}^{\left(2^{k} r_{B}\right)^{2}}\left|t \int_{0}^{r_{B}^{2}} \frac{1}{(t+s)^{2}} \Psi_{t, s}(\mathcal{L}) g(x) \chi_{T\left(2^{k} B\right) \backslash T\left(2^{k-1} B\right)}(x, t) d s\right|^{2} d t\right\}^{1 / 2}\right\|_{L^{2}\left(2^{k} B\right)} \\
\leq C \|\left\{\int_{0}^{\left(2^{k} r_{B}\right)^{2}} \mid t \int_{0}^{r_{B}^{2}} \int_{B\left(x_{B}, r_{B}\right)} \frac{1}{(t+s)^{n / 2+2}} e^{-c|x-y|^{2} /(t+s)}\right. \\
\left.\times\left.|g(y)| \chi_{T\left(2^{k} B\right) \backslash T\left(2^{k-1} B\right)}(x, t) d y d s\right|^{2} d t\right\}^{1 / 2} \|_{L^{2}\left(2^{k} B\right)} .
\end{gathered}
$$

Note that for $(x, t) \in T\left(2^{k} B\right) \backslash T\left(2^{k-1} B\right)$ and $y \in B$, we have that $|x-y| \geq 2^{k} r_{B}$. So

$$
\begin{aligned}
\mathrm{B}_{k} & \leq C\left\|\left\{\int_{0}^{\left(2^{k} r_{B}\right)^{2}}\left|t \int_{0}^{r_{B}^{2}} \int_{B\left(x_{B}, r_{B}\right)} \frac{1}{|x-y|^{n+4}}\right| g(y)\left|\chi_{T\left(2^{k} B\right) \backslash T\left(2^{k-1} B\right)}(x, t) d y d s\right|^{2} d t\right\}^{1 / 2}\right\|_{L^{2}\left(2^{k} B\right)} \\
& \leq C\|g\|_{L^{1}(B)}\left\|\left\{\int_{0}^{\left(2^{k} r_{B}\right)^{2}}\left|t \int_{0}^{r_{B}^{2}} \frac{1}{\left(2^{k} r_{B}\right)^{n+4}} \chi_{T\left(2^{k} B\right) \backslash T\left(2^{k-1} B\right)}(x, t) d s\right|^{2} d t\right\}^{1 / 2}\right\| \|_{L^{2}\left(2^{k} B\right)} \\
& \leq C\|g\|_{L^{1}(B)} \frac{r_{B}^{2}}{\left(2^{k} r_{B}\right)^{n+4}}\left\|\int_{0}^{\left(2^{k} r_{B}\right)^{2}} \chi_{T\left(2^{k} B\right) \backslash T\left(2^{k-1} B\right)}(x, t) t^{2} d t\right\|_{L^{1}\left(2^{k} B\right)}^{1 / 2} \\
& \leq C 2^{(-2-n / 2) k} r_{B}^{-n / 2-2}\|g\|_{L^{1}(B)} \leq C 2^{(-n / 2-2) k}\|g\|_{L^{2 n /(n+2)}(B)} .
\end{aligned}
$$

Consequently,

$$
\mathrm{A}_{k} \leq C 2^{-2 k} r_{B}^{\lambda / 2}\|\| \mu_{\nabla_{t}, f}\|\|_{(2, \lambda) \operatorname{car}}\|g\|_{L^{2 n /(n+2)(B)}}
$$

which implies

$$
\begin{aligned}
\int_{\mathbb{R}_{+}^{n+1}}|F(x, t) G(x, t)| \frac{d x d t}{t} \leq & C r_{B}^{\lambda / 2} \mid\left\|\mu_{\nabla_{t}, f}\right\|\left\|_{(2, \lambda) \operatorname{car}}\right\| g \|_{L^{2 n /(n+2)}(B)} \\
& +C \sum_{k=2}^{\infty} 2^{-2 k} r_{B}^{\lambda / 2}\|\| \mu_{f}\|\|_{(2, \lambda) \text { car }}\|g\|_{L^{2 n /(n+2)}(B)} \\
\leq & C r_{B}^{\lambda / 2} \mid\left\|\mu_{\nabla_{t}, f}\right\|\left\|_{(2, \lambda) \operatorname{car}}\right\| g \|_{L^{2 n /(n+2)}(B)}
\end{aligned}
$$


as desired.

Lemma 3.6. Suppose B, $f, g, F, G$ are defined as in Lemma 3.5. If \|\|$\mu_{\nabla_{t}, f}\|\|_{(2, \lambda) \text { car }}<$ $\infty$, then we have the equality

$$
\int_{\mathbb{R}^{n}} f(x)\left(\mathcal{I}-e^{-r_{B}^{2} \mathcal{L}}\right) g(x) d x=\frac{1}{4} \int_{\mathbb{R}_{+}^{n+1}} F(x, t) G(x, t) \frac{d x d t}{t} .
$$

Proof. The technique of this lemma's proof has been used in lots of papers, for example 2, 4, 6, 16, but it is notable to state it here for completeness.

By Lemma 3.5, we know that $\int_{\mathbb{R}_{+}^{n+1}}|F(x, t) G(x, t)| \frac{d x d t}{t}<\infty$. By dominated convergence theorem, the following integral converges absolutely and satisfies

$$
I=\int_{\mathbb{R}_{+}^{n+1}} F(x, t) G(x, t) \frac{d x d t}{t}=\lim _{\epsilon \rightarrow 0^{+}} \int_{\epsilon}^{1 / \epsilon} \int_{\mathbb{R}^{n}} F(x, t) G(x, t) \frac{d x d t}{t} .
$$

By Fubini's theorem, together with the commutative property of the semigroup $\left\{e^{-t \mathcal{L}}\right\}_{t>0}$, we have

$$
\int_{\mathbb{R}^{n}} F(x, t) G(x, t) d x=\int_{\mathbb{R}^{n}} f(y)\left(t \partial_{t} e^{-t \mathcal{L}}\right)^{2}\left(\mathcal{I}-e^{-r_{B}^{2} \mathcal{L}}\right) g(y) d y .
$$

Whence,

$$
\begin{aligned}
I & =\lim _{\epsilon \rightarrow 0^{+}} \int_{\epsilon}^{1 / \epsilon} \int_{\mathbb{R}^{n}} f(x)\left(t \partial_{t} e^{-t \mathcal{L}}\right)^{2}\left(\mathcal{I}-e^{-r_{B}^{2} \mathcal{L}}\right) g(x) \frac{d x d t}{t} \\
& =\lim _{\epsilon \rightarrow 0^{+}} \int_{\mathbb{R}^{n}} f(x) \int_{\epsilon}^{1 / \epsilon}\left(t \partial_{t} e^{-t \mathcal{L}}\right)^{2}\left(\mathcal{I}-e^{-r_{B}^{2} \mathcal{L}}\right) g(x) \frac{d t d x}{t} .
\end{aligned}
$$

By [6, Lemma 7], we can pass the limit inside the integral above. And, by a similar computation of [16, Lemma 3.7] with $\beta=1$, we have

$$
I=\int_{\mathbb{R}^{n}} f(x) \int_{0}^{\infty}\left(t \partial_{t} e^{-t \mathcal{L}}\right)^{2}\left(\mathcal{I}-e^{-r_{B}^{2} \mathcal{L}}\right) g(x) \frac{d t d x}{t}=\frac{1}{4} \int_{\mathbb{R}^{n}} f(x)\left(\mathcal{I}-e^{-r_{B}^{2} \mathcal{L}}\right) g(x) d x
$$

This completes the proof.

Now, we are in a position to prove Lemma 3.4 .

Proof of Lemma 3.4. First, we note an equivalent characterization of $\mathrm{L}^{2, \lambda}\left(\mathbb{R}^{n}\right)$ that $f \in$ $\mathrm{L}^{2, \lambda}\left(\mathbb{R}^{n}\right)$ if and only if $f \in L^{2}\left((1+|x|)^{-(n+\epsilon)} d x\right)$ and

$$
\sup _{B}\left(|B|^{-\lambda / n} \int_{B}\left|f(x)-e^{-r_{B}^{2} \mathcal{L}} f(x)\right|^{2} d x\right)^{1 / 2} \leq C<\infty .
$$

This has been proved in [4, Proposition 6.11] (see also [3, 13, 20]). 
Now if $\|u\|_{\mathrm{TL}_{\mathcal{L}}^{\lambda}\left(\mathbb{R}_{+}^{n+1}\right)}<\infty$, then it follows from Lemma 3.1 that

$$
\int_{\mathbb{R}^{n}} \frac{\left|f_{k}(x)\right|^{2}}{1+|x|^{2 n}} d x \leq C_{k}<\infty
$$

Given an $L^{2}$ function $g$ supported on a ball $B=B\left(x_{B}, r_{B}\right)$, it follows from Lemma 3.6 that we have

$$
\int_{\mathbb{R}^{n}} f_{k}(x)\left(I-e^{-r_{B}^{2} \mathcal{L}}\right) g(x) d x=\frac{1}{4} \int_{\mathbb{R}_{+}^{n+1}} t \partial_{t} e^{-t \mathcal{L}} f_{k}(x) t \partial_{t} e^{-t \mathcal{L}}\left(\mathcal{I}-e^{-r_{B}^{2} \mathcal{L}}\right) g(x) \frac{d x d t}{t} .
$$

By Lemmas 3.3 and 3.5 .

$$
\begin{aligned}
\left|\int_{\mathbb{R}^{n}} f_{k}(x)\left(\mathcal{I}-e^{-r_{B}^{2} \mathcal{L}}\right) g(x) d x\right| & \leq C|B|^{\lambda /(2 n)}\|\| \mu_{\nabla_{t}, f_{k}} \mid\left\|_{(2, \lambda) \operatorname{car}}\right\| g \|_{L^{2 n /(n+2)}(B)} \\
& \leq C|B|^{\lambda /(2 n)}\|u\|_{\mathrm{TL}_{\mathcal{L}}^{\lambda}\left(\mathbb{R}_{+}^{n+1}\right)}\|g\|_{L^{2 n /(n+2)}(B)} .
\end{aligned}
$$

Then the duality argument for $L^{2}$ shows that

$$
\begin{aligned}
& \left(|B|^{-\lambda / n} \int_{B}\left|f_{k}(x)-e^{-r_{B}^{2} \mathcal{L}} f_{k}(x)\right|^{2} d x\right)^{1 / 2} \\
= & |B|^{-\lambda /(2 n)} \sup _{\|g\|_{L^{2}(B)} \leq 1}\left|\int_{\mathbb{R}^{n}}\left(\mathcal{I}-e^{-r_{B}^{2} \mathcal{L}}\right) f_{k}(x) g(x) d x\right| \\
\leq & |B|^{-\lambda /(2 n)} \sup _{\|g\|_{L^{2 n /(n+2)(B)}} \leq 1}\left|\int_{\mathbb{R}^{n}} f_{k}(x)\left(\mathcal{I}-e^{-r_{B}^{2} \mathcal{L}}\right) g(x) d x\right| \\
\leq & C\|u\|_{\mathrm{TL}_{\mathcal{L}}^{\lambda}\left(\mathbb{R}_{+}^{n+1}\right)}
\end{aligned}
$$

for some $C>0$ independent of $k$.

It then follows that for all $k \in \mathbb{N},\left\{f_{k}\right\}$ is uniformly bounded in $\mathrm{TL}_{\mathcal{L}}^{\lambda}\left(\mathbb{R}_{+}^{n+1}\right)$.

Proof of Theorem 1.2(1). Recall that the condition $V \in B_{n}$ implies $V \in B_{q_{0}}$ for some $q_{0}>n / 2$. From Lemmas 2.1 and 2.2 , we see that $u(x, t)=e^{-t \mathcal{L}} f(x) \in C^{1}\left(\mathbb{R}_{+}^{n+1}\right)$. It will be enough to finish the proof if we have proved

$$
\|u\|_{\mathrm{TL}_{\mathcal{L}}^{\lambda}\left(\mathbb{R}_{+}^{n+1}\right)} \leq C\|f\|_{L^{2, \lambda}\left(\mathbb{R}^{n}\right)} .
$$

To prove (3.9), by a similar argument in 6 , we can easily prove that for every $f \in L^{2, \lambda}\left(\mathbb{R}^{n}\right)$, the term $\left|\partial_{t} e^{-t \mathcal{L}}(f)(x)\right|^{2}$ has the following estimate (see [6, Theorem 2]):

$$
\sup _{x_{B}, r_{B}} r_{B}^{-\lambda} \int_{0}^{r_{B}^{2}} \int_{B\left(x_{B}, r_{B}\right)}\left|\partial_{t} e^{-t \mathcal{L}} f(x)\right|^{2} d x d t \leq\|f\|_{L^{2, \lambda}\left(\mathbb{R}^{n}\right)} .
$$


So, we only need to estimate the term $\left|\nabla_{x} e^{-t \mathcal{L}}(f)(x)\right|^{2}$. In fact

$$
\begin{aligned}
\left(\frac{1}{r_{B}^{\lambda}} \int_{0}^{r_{B}^{2}} \int_{B}\left|\nabla_{x} e^{-t \mathcal{L}} f(x)\right|^{2} d x d t\right)^{1 / 2} & \leq \sum_{k=0}^{\infty} \frac{1}{r_{B}^{\lambda / 2}}\left(\int_{0}^{r_{B}^{2}} \int_{B}\left|\nabla_{x} e^{-t \mathcal{L}} f_{k}(x)\right|^{2} d x d t\right)^{1 / 2} \\
& =: \sum_{k=0}^{\infty} J_{k}
\end{aligned}
$$

where $f_{0}=f \chi_{2 B}$ and $f_{k}=f \chi_{2^{k+1} B \backslash 2^{k} B}$ for $k \in \mathbb{N}^{+}$. For $J_{0}$, since the Riesz transform $\nabla \mathcal{L}^{-1 / 2}$ is bounded on $L^{2}\left(\mathbb{R}^{n}\right)$, by $(3.8)$ and the commutative property of $e^{-\mathcal{L}}$ and $\mathcal{L}^{-1 / 2}$, we have

$$
\begin{aligned}
J_{0}^{2} & =\frac{1}{r_{B}^{\lambda}} \int_{0}^{r_{B}^{2}} \int_{B}\left|\nabla_{x} e^{-t \mathcal{L}} f_{0}(x)\right|^{2} d x d t \\
& \leq \frac{1}{r_{B}^{\lambda}} \int_{0}^{r_{B}^{2}} \int_{\mathbb{R}^{n}}\left|\nabla_{x} \mathcal{L}^{-1 / 2} \mathcal{L}^{1 / 2} e^{-t \mathcal{L}} f_{0}(x)\right|^{2} d x d t \\
& \leq C \frac{1}{r_{B}^{\lambda}} \int_{0}^{\infty} \int_{\mathbb{R}^{n}}\left|\partial_{t} e^{-t \mathcal{L}}\left(\mathcal{L}^{-1 / 2} f_{0}\right)(x)\right|^{2} d x d t \\
& \leq C \frac{1}{r_{B}^{\lambda}}\left\|\mathcal{L}^{1 / 2} \mathcal{L}^{-1 / 2} f_{0}\right\|_{L^{2}\left(\mathbb{R}^{n}\right)}^{2}=C \frac{1}{r_{B}^{\lambda}} \int_{2 B}|f(x)|^{2} d x \\
& \leq C\|f\|_{L^{2, \lambda}\left(\mathbb{R}^{n}\right)}^{2}
\end{aligned}
$$

When $k \geq 1$, for any $x \in B$ and $k \in \mathbb{N}^{+}$, we apply Lemma 2.2(i) to obtain

$$
\begin{aligned}
\left|\nabla_{x} e^{-t \mathcal{L}} f_{k}(x)\right| & \leq C \int_{2^{k+1} B / 2^{k} B} t^{-(n+1) / 2} e^{-|x-y|^{2} /(c t)}|f(y)| d y \\
& \leq C \int_{2^{k+1} B / 2^{k} B}|x-y|^{-(n+1)}|f(y)| d y \\
& \leq C \frac{1}{\left(2^{k} r_{B}\right)^{n+1}} \int_{2^{k+1} B}|f(y)| d y \\
& \leq C \frac{1}{\left(2^{k} r_{B}\right)^{1+(n-\lambda) / 2}}\|f\|_{L^{2, \lambda}\left(\mathbb{R}^{n}\right)}
\end{aligned}
$$

which yields

$$
\left|J_{k}\right| \leq C 2^{-k(1+(n-\lambda) / 2)}\|f\|_{L^{2, \lambda}\left(\mathbb{R}^{n}\right)} .
$$

Hence $\sum_{k=0}^{\infty}\left|J_{k}\right| \leq C\|f\|_{L^{2, \lambda}\left(\mathbb{R}^{n}\right)}$, and then $\|u\|_{\mathrm{TL}_{\mathcal{L}}^{\lambda}\left(\mathbb{R}^{n+1}\right)} \leq C\|f\|_{L^{2, \lambda}\left(\mathbb{R}^{n}\right)}$.

Proof of Theorem 1.2(2). To prove it, we will use the argument as in 45,7,15] and apply the key Lemma 3.4. Suppose $u \in \operatorname{TL}_{\mathcal{L}}^{\lambda}\left(\mathbb{R}_{+}^{n+1}\right)$, our aim is to find a function $f \in L^{2, \lambda}\left(\mathbb{R}^{n}\right)$ such that

$$
u(x, t)=e^{-t \mathcal{L}} f(x) \text { for each }(x, t) \in \mathbb{R}_{+}^{n+1} .
$$


To do this, for every $k \in \mathbb{N}^{+}$, we write $f_{k}=u(x, 1 / k)$. By Lemma 3.4, we obtain

$$
\int_{B\left(0,2^{j}\right)}\left|f_{k}(x)\right|^{2} d x \leq C 2^{j \lambda}\|u\|_{\mathrm{TL}_{\mathcal{L}}^{\lambda}\left(\mathbb{R}_{+}^{n+1}\right)}^{2} .
$$

This means that the sequence $\left\{f_{k}\right\}_{k=1}^{\infty}$ is bounded in $L^{2}\left(B\left(0,2^{j}\right)\right)$. So by passing to a subsequence, the sequence $\left\{f_{k}\right\}$ converges weakly to a function $g_{j} \in L^{2}\left(B\left(0,2^{j}\right)\right)$. Then, for $i>j$, we can get $g_{i}(x)=g_{j}(x)$, for almost everywhere $x \in B\left(0,2^{j}\right)$. Next, we define a function $f(x)$ by

$$
f(x)=g_{j}(x) \quad \text { if } x \in B\left(0,2^{j}\right), j=1,2,3, \ldots
$$

It is easy to see that $f$ is well defined on $\mathbb{R}^{n}=\bigcup_{j=1}^{\infty} B\left(0,2^{j}\right)$ and (after passing to a subsequence) $f_{k} \rightarrow f$ in $L^{2}$ on every ball of $\mathbb{R}^{n}$. It is also easy to check that for any open ball $B \subset \mathbb{R}^{n}$, we have

which implies

$$
\int_{B}|f(x)|^{2} d x \leq C r_{B}^{\lambda}\|u\|_{\mathrm{TL}_{\mathcal{L}}^{\lambda}\left(\mathbb{R}_{+}^{n+1}\right)}^{2}
$$

$$
\|f\|_{L^{2, \lambda}\left(\mathbb{R}^{n}\right)} \leq C\|u\|_{\mathrm{TL}_{\mathcal{L}}^{\lambda}\left(\mathbb{R}_{+}^{n+1}\right)}
$$

Finally, we will show that $u(x, t)=e^{-t \mathcal{L}} f(x)$. Since $u(x, \cdot)$ is continuous on $\mathbb{R}_{+}$, we have $u(x, t)=\lim _{k \rightarrow+\infty} u(x, t+1 / k)$. Then we have $u(x, t)=\lim _{k \rightarrow+\infty} e^{-t \mathcal{L}}(u(\cdot, 1 / k))(x)$. It reduces to show

$$
\lim _{k \rightarrow+\infty} e^{-t \mathcal{L}}(u(\cdot, 1 / k))(x)=e^{-t \mathcal{L}} f(x) .
$$

Indeed, we recall that $\mathcal{H}_{t}(x, t)$ is the kernel of $e^{-t \mathcal{L}}$. Then for any $l \in \mathbb{N}$, we write

$$
e^{-t \mathcal{L}}(u(\cdot, 1 / k))(x)=\int_{B\left(x, 2^{l} t\right)} \mathcal{H}_{t}(x, y) f_{k}(y) d y+\int_{B\left(x, 2^{l} t\right)^{c}} \mathcal{H}_{t}(x, y) f_{k}(y) d y .
$$

By Lemma 2.1 and the Hölder inequality, we have

$$
\begin{aligned}
\left|\int_{\left(B\left(x, 2^{l} t\right)\right)^{c}} \mathcal{H}_{t}(x, y) f_{k}(y) d y\right| & \leq C \sum_{i=l}^{\infty} \int_{B\left(x, 2^{i+1} t\right) / B\left(x, 2^{i} t\right)} t^{-n / 2} e^{-|x-y|^{2} /(c t)}\left|f_{k}(y)\right| d y \\
& \leq C \sum_{i=l}^{\infty}\left(2^{i} t\right)^{-n} \int_{B\left(x, 2^{i+1} t\right)}\left|f_{k}(y)\right| d y \\
& \leq C \sum_{i=l}^{\infty}\left(2^{i} t\right)^{(\lambda-n) / 2}\left\|f_{k}\right\|_{L^{2, \lambda}\left(\mathbb{R}^{n}\right)} \\
& \leq C 2^{-l(n-\lambda) / 2} t^{(\lambda-n) / 2}\left\|f_{k}\right\|_{L^{2, \lambda}\left(\mathbb{R}^{n}\right)} .
\end{aligned}
$$

By Lemma 3.4, we have that $\left\|f_{k}\right\|_{L^{2, \lambda}\left(\mathbb{R}^{n}\right)} \leq C\|u\|_{\mathrm{TL}_{\mathcal{L}}^{\lambda}\left(\mathbb{R}_{+}^{n+1}\right)}$ for some constant $C>0$ independent of $k$. Since $\lambda \in(0, n)$, we have

$$
\begin{aligned}
& \limsup _{l \rightarrow+\infty} \limsup _{k \rightarrow+\infty}\left|\int_{B\left(x, 2^{l} t\right)^{c}} \mathcal{H}_{t}(x, y) f_{k}(y) d y\right| \\
\leq & \lim _{l \rightarrow+\infty}\left(C 2^{-l((n-\lambda) / 2)} t^{(\lambda-n) / 2}\|u\|_{\mathrm{TL}_{\mathcal{L}}^{\lambda}\left(\mathbb{R}_{+}^{n+1}\right)}\right)=0 .
\end{aligned}
$$


Therefore,

$$
\lim _{k \rightarrow+\infty} e^{-t \mathcal{L}}\left(u\left(\cdot, k^{-1}\right)\right)(x)=\lim _{k \rightarrow+\infty} \lim _{l \rightarrow+\infty} \int_{B\left(x, 2^{l} t\right)} \mathcal{H}_{t}(x, y) f_{k}(y) d y=e^{-t \mathcal{L}} f(x) .
$$

We have showed that $u(x, t)=e^{-t \mathcal{L}} f(x)$. The proof of Theorem 1.2 is completed.

\section{Acknowledgments}

This research was partly supported by the Natural Science Foundation of Zhejiang Province (Grant No. LY18A010006 and No. LQ18A010005) and the National Natural Science Foundation of China (Grant No. 11401525 and No. 11801518).

The authors would like to thank the referee's professional comments which improved our results in this paper.

\section{References}

[1] M. Dindos, C. Kenig and J. Pipher, BMO solvability and the $A_{\infty}$ condition for elliptic operators, J. Geom. Anal. 21 (2011), no. 1, 78-95.

[2] X. T. Duong, J. Xiao and L. Yan, Old and new Morrey spaces with heat kernel bounds, J. Fourier Anal. Appl. 13 (2007), no. 1, 87-111.

[3] X. T. Duong and L. Yan, New function spaces of BMO type, the John-Nirenberg inequality, interpolation, and applications, Comm. Pure Appl. Math. 58 (2005), no. 10, 1375-1420.

[4] Duality of Hardy and BMO spaces associated with operators with heat kernel bounds, J. Amer. Math. Soc. 18 (2005), no. 4, 943-973.

[5] X. T. Duong, L. Yan and C. Zhang, On characterization of Poisson integrals of Schrödinger operators with BMO traces, J. Funct. Anal. 266 (2014), no. 4, 20532085.

[6] J. Dziubański, G. Garrigós, T. Martínez, J. L. Torrea and J. Zienkiewicz, BMO spaces related to Schrödinger operators with potentials satisfying a reverse Hölder inequality, Math. Z. 249 (2005), no. 2, 329-356.

[7] E. B. Fabes, R. L. Johnson and U. Neri, Spaces of harmonic functions representable by Poisson integrals of functions in $B M O$ and $L_{p, \lambda}$, Indiana Univ. Math. J. 25 (1976), no. $2,159-170$. 
[8] E. B. Fabes and U. Neri, Characterization of temperatures with initial data in BMO, Duke Math. J. 42 (1975), no. 4, 725-734.

[9] _ Dirichlet problem in Lipschitz domains with BMO data, Proc. Amer. Math. Soc. 78 (1980), no. 1, 33-39.

[10] C. Fefferman and E. M. Stein, $H^{p}$ spaces of several variables, Acta Math. 129 (1972), no. 3-4, 137-193.

[11] W. Gao and Y. Jiang, $L^{p}$ estimate for parabolic Schrödinger operator with certain potentials, J. Math. Anal. Appl. 310 (2005), no. 1, 128-143.

[12] F. W. Gehring, The $L^{p}$-integrability of the partial derivatives of a quasiconformal mapping, Acta Math. 130 (1973), 265-277.

[13] S. Hofmann, G. Lu, D. Mitrea, M. Mitrea and L. Yan, Hardy spaces associated to non-negative self-adjoint operators satisfying Davies-Gaffney estimates, Mem. Amer. Math. Soc. 214 (2011), no. 1007, 78 pp.

[14] S. Hofmann, S. Mayboroda and M. Mourgoglou, Layer potentials and boundary value problems for elliptic equations with complex $L^{\infty}$ coefficients satisfying the small Carleson measure norm condition, Adv. Math. 270 (2015), 480-564.

[15] R. Jiang, J. Xiao and D. Yang, Towards spaces of harmonic functions with traces in square Campanato spaces and their scaling invariants, Anal. Appl. (Singap.) 14 (2016), no. 5, 679-703.

[16] T. Ma, P. R. Stinga, J. L. Torrea and C. Zhang, Regularity properties of Schrödinger operators, J. Math. Anal. Appl. 388 (2012), no. 2, 817-837.

[17] _ Regularity estimates in Hölder spaces for Schrödinger operators via a T1 theorem, Ann. Mat. Pura Appl. (4) 193 (2014), no. 2, 561-589.

[18] C. B. Morrey, On the solutions of quasi-linear elliptic partial differential equations, Trans. Amer. Math. Soc. 43 (1938), no. 1, 126-166.

[19] Z. Shen, $L^{p}$ estimates for Schrödinger operators with certain potentials, Ann. Inst. Fourier (Grenoble) 45 (1995), no. 2, 513-546.

[20] L. Song, X. X. Tian and L. X. Yan, On characterization of Poisson integrals of Schrödinger operators with Morrey traces, Acta Math. Sin. (Engl. Ser.) 34 (2018), no. 4, 787-800. 
[21] E. M. Stein, Topics in Harmonic Analysis Related to the Littlewood-Paley Theory, Annals of Mathematics Studies 63, Princeton University Press, Princeton, N.J., 1970.

[22] E. M. Stein and G. Weiss, Introduction to Fourier Analysis on Euclidean Spaces, Princeton Mathematical Series 32, Princeton University Press, Princeton, N.J., 1971.

[23] L. Tang and J. Han, $L^{p}$ boundedness for parabolic Schrödinger type operators with certain nonnegative potentials, Forum Math. 23 (2011), no. 1, 161-179.

[24] L. Wu and L. Yan, Heat kernels, upper bounds and Hardy spaces associated to the generalized Schrödinger operators, J. Funct. Anal. 270 (2016), no. 10, 3709-3749.

[25] M. Yang and C. Zhang, Characterization of temperatures associated to Schrödinger operators with initial data in BMO spaces, arXiv:1710.01160.

[26] W. Yuan, W. Sickel and D. Yang, Morrey and Campanato meet Besov, Lizorkin and Triebel, Lecture Notes in Mathematics 2005, Springer-Verlag, Berlin, 2010.

Qiang Huang

Department of Mathematics, Zhejiang Normal University, Jinhua 321004, China

E-mail address: huangqiang0704@163.com

Chao Zhang

School of Statistics and Mathematics, Zhejiang Gongshang University, Hangzhou 310018, China

E-mail address: zaoyangzhangchao@163.com 